\title{
ON THE EXISTENCE OF CHAPMAN-JOUGUET DETONATION WAVES
}

\author{
Mahmoud Hesaaraki and Abdolrahman Razani
}

\begin{abstract}
The existence of travelling wave solutions to equations of a viscous, heat-conducting combustible fluid is proved. The reactions are assumed to be one step exothermic reactions with a natural discontinuous reaction rate function. The problem is studied for a general gas. Instead of assuming the ideal gas conditions we consider a general thermodynamics which is described by a fairly mild set of hypotheses. The existence proof of travelling waves for Chapman-Jouguet detonation reduces to finding specific heteroclinic orbits of a discontinuous system of ordinary differential equations; these heteroclinic orbits connect a rest point corresponding to unburnt state to that of the burnt state. The existence proof for heteroclinic orbits corresponding to ChapmanJouguet detonation waves is carried out by some general topological arguments in ordinary differential equations theory.
\end{abstract}

\section{INTRODUCTION}

Travelling wave problems for plane detonation waves which are compressive, exothermically reacting shock waves, have been studied by many authors, for example Wagner [10], Gardner [1], Gasser and Szmolyan [2]. In the present paper we shall discuss the existence of travelling waves as well as the existence of structure for Chapman-Jouguet detonations, for gases governed by general equations of state without having any special assumption on the reaction rate function. When these equations, for a one step reaction, are specialised to a single space variable with a simplified reactant-product to kinetic mechanism, they have the following form in Eulerian coordinates:

$$
\begin{aligned}
\rho_{t}+(\rho u)_{x} & =0 \\
(\rho u)_{t}+\left(\rho u^{2}+p\right)_{x} & =\left(\nu u_{x}\right)_{x}, \\
\left(\frac{1}{2} \rho u^{2}+\rho e\right)_{t}+\left(u\left(\frac{1}{2} \rho u^{2}+\rho e\right)\right)_{x}+(p u)_{x} & =\left(\nu u_{x} u\right)_{x}+\left(\lambda T_{x}\right)_{x}+D\left(\left(h_{0}-h_{1}\right) \rho Y_{x}\right)_{x} \\
(\rho Y)_{t}+(\rho u Y)_{x} & =-K \Phi(T) \rho Y+D\left(\rho Y_{x}\right)_{x} .
\end{aligned}
$$

Received 25th July, 2000

Both authors would like to thank the Institute for Studies in Theoretical Physics and Mathematics,IPM, Tehran, Iran for supporting this research.

Copyright Clearance Centre, Inc. Serial-fee code: 0004-9727/01 \$A2.00+0.00. 
Here $\rho$ is the density, $u$ the velocity of the flow in the direction of the $x$-axis, $e$ the absolute internal energy per unit mass of the mixture, $p$ the pressure, $T$ the temperature, $Y$ the mass fraction of the unburnt gas, and $h_{0}$ and $h_{1}$ are the specific enthalpies of the reactant and the product, respectively. The parameters $\nu, \lambda, K$, and $D$ are the bulk viscosity, the heat transfer coefficient, the reaction rate coefficient, and the diffusion rate for the reactant, respectively. These parameters are always nonnegative. The independent variables $t$ and $x$ are the time variable and a space variable, respectively. The function $\Phi(T)$ which is called the "reaction rate function" is defined by:

$$
\Phi(T)= \begin{cases}0 & \text { for } T<T_{i}, \\ \Phi_{1}(T) & \text { for } T \geqslant T_{i},\end{cases}
$$

where $\Phi_{1}(T)=T^{\gamma} e^{-A / T}$ (for some positive constants $\gamma$ and $A$ ), is a smooth positive function and $T_{i}$ is the "ignition temperature" of the reaction.

Finally, if the absolute internal energy and pressure of the unburnt and burnt gases are denoted by $e_{0}, e_{1}, p_{0}$, and $p_{1}$, respectively, then

$$
e=Y e_{0}+(1-Y) e_{1} \text { and } p=Y p_{0}+(1-Y) p_{1},
$$

are the absolute internal energy and pressure of the mixture gas, respectively. Moreover, from the thermodynamical point of view, in the case of exothermic reactions we have

$$
e_{0}>e_{1}, p_{0}<p_{1} \text {, and } h_{0}>h_{1} \text {. }
$$

For details and the derivations of the above equations, we refer the readers to [10].

- The rest of the paper is organised as follows. In Section 2, we introduce the hypotheses and the problem, and then make some observations related to the problem. In Section 3 we shall consider two general existence theorems in ordinary differential equation theory related to the problem. In Section 4 we shall show that travelling wave solutions for Chapman-Jouguet detonation waves exist.

\section{THE HYPOTHESES AND THE PROBLEM}

Travelling waves are solutions of (1.1) depending only on $\xi=x-s t$, which connect an unburnt state at $\xi=-\infty$ to a burnt state at $\xi=+\infty$, [8]. The parameter $s(\in \mathbb{R}$ ) (which is called the speed of the combustion wave) satisfies "the jump and entropy conditions". Hence for getting travelling wave solutions of (1.1), the system (1.1) reduces to the following system of equations:

$$
-s \rho_{\xi}+(\rho u)_{\xi}=0
$$

$$
\begin{aligned}
-s\left(\frac{1}{2} \rho u^{2}+\rho e\right)_{\xi}+\left(u\left(\frac{1}{2} \rho u^{2}+\rho e\right)\right)_{\xi}+(p u)_{\xi} & =\left(\nu u_{\xi} u\right)_{\xi}+\left(\lambda T_{\xi}\right)_{\xi}+\left(D\left(h_{0}-h_{1}\right) \rho Y_{\xi}\right)_{\xi} \\
-s(\rho Y)_{\xi}+(\rho u Y)_{\xi} & =-K \Phi(T) \rho Y+D\left(\rho Y_{\xi}\right)_{\xi} .
\end{aligned}
$$


The first equation of (2.1) can be integrated once to give $-s \rho+\rho u=a$, where without loss of generality, we may assume $a=1$. Set $V=1 / \rho$, the specific volume of the gas, Then $u=V+s$. Let $Z=-Y+D \rho Y_{\xi}$ be an auxiliary variable. Also in order to avoid confusion in notation and to take advantage of some results from our previous works $[3,4,5,6,7]$, we replace $\nu, \xi, \lambda, D, K, h_{0}-h_{1}$ and $Y$ by $\eta, t, k, \alpha, \beta^{-1}, q$ and $1-X$. By using these relations, the system (2.1) becomes:

$$
\begin{aligned}
\eta \dot{V} & =V-J+p:=G_{1}(u), \\
k \dot{T} & =e-\frac{1}{2} V^{2}+J V-C-q(1-X+Z):=G_{2}(u), \\
\alpha \dot{X} & =-V(1-X+Z):=G_{3}(u), \\
\beta V \dot{Z} & =(1-X) \Phi(T):=G_{4}(u),
\end{aligned}
$$

where "." means $\frac{d}{d t}$ and $u=(V, T, X, Z)^{T}$. Also the system (2.2) can be written as:

$$
A \dot{u}=G(u),
$$

where $A=\operatorname{diag}(\eta, k, \alpha, \beta V)$ and $G(u)=\left(G_{1}(u), G_{2}(u), G_{3}(u), G_{4}(u)\right)^{T}$.

Let $S(V, T, X)$ be the entropy of the system. We assume that $e(V, T, X), p(V, T, X)$ and $S(V, T, X)$ satisfy the Hypotheses $H_{1}-H_{5}$ in $[3,4,5,6,7,8]$ and $H_{6}-H_{8}$ below [11].

$\left(H_{6}\right)$ The Gibb's law of thermodynamics is given by $d e=T d S-p d V+$ $\left(h_{1}-h_{0}\right) d X$, where $h_{i}$, for $i=0,1$, is as before.

$\left(H_{7}\right)$ The following identities hold: $e_{i V}=T S_{i V}-p_{i}, e_{i T}=T S_{i T}, p_{i T}=S_{i V}$ and $h_{i}=e_{i}+p_{i} V$. Moreover, $S_{i T}>0$ for $i=0,1$, where $S_{i}=S(V, T, i)$.

$\left(H_{8}\right)$ Consider $q$ as a function of $V$ and $T, q_{V} \geqslant 0$ and $q_{T} \geqslant 0$.

We shall use these Hypotheses directly, or shall take advantage of some results from previous work based on them.

For fixed positive viscosity parameters $\eta, k, \alpha$ and $\beta$, we are looking for some orbits of (2.2) which are defined for all $t \in \mathbb{R}$ and connect two different rest points of this system of ordinary differential equations. Therefore in the first step we must determine the rest points of this system. In order to do this, we must have $G(u)=0$, at a rest point. Since $\Phi(T)=0$ for $T<T_{i}$, and this set is contained in the region $0 \leqslant X<1$, thus from $G_{4}(u)=0$, at a rest point, we must have $X=1$ or $T<T_{i}$.

CASE 1. $X=1$. We arrive at the following criterion at a rest point:

$$
\begin{aligned}
& F_{11}(V, T)=V-J+p_{1}(V, T)=0, \\
& F_{12}(V, T)=e_{1}(V, T)-\frac{1}{2} V^{2}+J V-C=0 .
\end{aligned}
$$


CASE 2. $\Phi(T)=0$. In this case, at the rest point we must have $T<T_{i}$, since $1-X+Z=$ 0 . By using this fact and setting $X=m$, we have:

$$
\begin{aligned}
& F_{m 1}(V, T)=V-J+p(V, T, m)=0, \\
& F_{m 2}(V, T)=e(V, T, m)-\frac{1}{2} V^{2}+J V-C=0 .
\end{aligned}
$$

With respect to Hypotheses $H_{5}$ and $H_{7}$, the equations $F_{m 1}(V, T)=0$ and $F_{m 2}(V, T)=$ 0 determine graphs of functions $T_{m 1}(V)$ and $T_{m 2}(V)$ in the $V, T$ plane, respectively.

The following Lemmas 2.1, 2.2 and 2.3 correspond to [4, Lemmas 2.1, 2.2. and 2.3], so their proofs are omitted. These lemmas give some information about the rest points of the system (2.2). See also [3, Section 3.2].

LEMMA 2.1. For $0 \leqslant m \leqslant 1, \frac{d T_{m 1}(V)}{d V}=0$ for precisely one value of $V$. This is a relative maximum.

LEMma 2.2. For fixed $J>0$, there is a number $C_{m} \in \mathbb{R}$ such that for $C>C_{m}$, the system of algebraic equations (2.5) (or (2.4)) admits no solutions. For $C=C_{m}$ it admits one solution, and for $C<C_{m}$ it admits two solutions, $0 \leqslant m \leqslant 1$.

Here we assume that $F_{m 1}(V, T)=0$ and $F_{m 2}(V, T)=0$ intersect each other at two points, say $\left(V_{m j}, T_{m j}\right), j=0,1$, with $V_{m 0}>V_{m 1}$, for $0 \leqslant m<1$. For $m=1$ we consider the case where these two rest points can coincide to each other. Using this notation, from [6, Corollary 2.1] we have the following two corollaries.

Corollary 2.1. The curve $\left\{(V, T): F_{m 2}(V, T)=0, V_{m 1}<V<V_{m 0}\right\}$ lies in the region $\left\{(V, T): F_{m 1}(V, T)<0\right\}$, for $0 \leqslant m \leqslant 1$.

Corollary 2.2. For $0 \leqslant m \leqslant 1$, we have $S\left(V_{m 0}, T_{m 0}, m\right)<S\left(V_{m 1}, T_{m 1}, m\right)$.

LEMMA 2.3. The function $T_{m 2}(V)$ is strictly decreasing in the interval $\left[V_{m 1}, V_{m 0}\right]$.

For the proofs of the above lemmas the reader is referred to [4].

The graphs of $F_{m 1}(V, T)=0$ and $F_{m 2}(V, T)=0$ are depicted in [6, Figure 2.1].

Let $\left(V_{m j}, T_{m j}\right), 0 \leqslant m<1, j=0,1$, and $\left(V_{1}, T_{1}\right)$ be as above. Here we assume

$$
T_{00}<T_{i}<T_{1}
$$

and we shall show that $T_{m 0}<T_{1}$ in Theorem 2.1.

By considering the above results the rest points of the system (2.2) are:

$$
\begin{aligned}
u_{1} & =\left(V_{1}, T_{1}, 1,0\right)^{T}, \\
u_{m 0} & =\left(V_{m 0}, T_{m 0}, m, m-1\right)^{T}, 0 \leqslant m<1, T_{m 0}<T_{i} .
\end{aligned}
$$

In the present work it is assumed that the rest point $u_{1}$ exists.

THEOREM 2.1. Let $V_{m j}$ and $T_{m j}, 0 \leqslant m<1, j=0$ and $\left(V_{1}, T_{1}\right)$ be as above. If (1.3) and (1.4) hold, then for $0 \leqslant m<n \leqslant 1$ we have $V_{m 0}>V_{1}>V_{m 1}$ and $T_{m 0}<T_{1}$. 
Proof: It is easy to see from [5, Corollary 2.1], that whenever $0 \leqslant m<n \leqslant 1$, then:

$$
\begin{aligned}
& T_{m 1}(V)=T_{1}(V, m)>T_{1}(V, n)=T_{n 1}(V), \\
& T_{m 2}(V)=T_{2}(V, m)<T_{2}(V, n)=T_{n 2}(V) .
\end{aligned}
$$

Hence $\left(V_{1}, T_{1}\right)$ is located in the domain $\left\{(V, T): \widetilde{G}_{1}(V, T, m)<0\right.$ and $\left.\tilde{G}_{2}(V, T, m)>0\right\}$. It then follows that $V_{m 0}>V_{1}>V_{m 1}$ and $T_{m 0}<T_{1}$.

COROLlary 2.3. If the rest point $u_{1}$ exists, then the rest point $u_{m 0}$ exists for some $0 \leqslant m<1$.

We shall use the above results about the rest points in Section 4.

In Section 4 we shall show that for the general discontinuous reaction rate function $\Phi(T)$ the travelling wave solutions for Chapman-Jouguet detonation waves exist.

\section{SOME EXISTENCE THEOREMS IN ORDINARY DIFFERENTIAL EQUATIONS}

In this section we shall prove some existence theorems in ordinary differential equation theory which will be used in the next section to prove the existence of travelling waves for Chapman-Jouguet detonation waves. Prior to them we consider

$$
\frac{d x}{d t}=f(x), x=\left(x_{1}, x_{2}, \cdots, x_{n}\right)^{T},
$$

as an autonomous system of ordinary differential equations in $\mathbb{R}^{n}$.

THEOREM 3.1. Suppose $f$ in (3.1) is locally Lipschitz in a neighbourhood of the closure of an open bounded set $D$ which is homeomorphic to the parabola $\left\{x \in \mathbb{R}^{n}\right.$ : $\left.\sum_{i=1}^{n-1} x_{i}^{2}<1-x_{n}, 0<x_{n}<1\right\}$, and (3.1) is gradient-like with respect to a real valued function $h$ in $D$. Moreover, the following conditions hold.

$C_{1}$ : The set $\{x \in \bar{D}: h(x)=c\}$ corresponds to the set $\left\{x \in \mathbb{R}^{n}: \sum_{i=1}^{n-1} x_{i}^{2} \leqslant\right.$ $\left.1-c_{n}, x_{n}=c\right\}$ for $c \in[0,1]$, under the homeomorphism.

$C_{2}$ : The set $\{x \in \bar{D}: h(x)=1\}$, which consists of a single point, say $\widetilde{x}$, is a rest point of (3.1), and $\tilde{x}$ is the only rest point of (3.1) in $\bar{D}$.

$C_{3}$ : Let $E=\{x \in \partial D: h(x)>0\}$. For $y \in \bar{E} \backslash\{\widetilde{x}\}$, y.t $\notin D$ for small positive $t$ and $y . t \notin \partial D$ for small $|t| \neq 0$.

$C_{4}$ : Let $F=\partial D \backslash \bar{E}$. For $y \in F$, y.t $\in D$ for $t>0$ and small.

Then there is point $p \in F$ such that p.t $\in D$ for $t>0$ and $\lim _{t \rightarrow+\infty} p . t=\widetilde{x}$.

ProOF: Suppose such a point $p$ does not exist. Then for each $y \in F$ there is $t>0$ such that $y . t \notin \bar{D}$. Then, by $C_{4}$, there is $t(y)>0$ such that $y \cdot(0, t(y)) \subset D, y . t(y) \in \bar{E}$ 
and $y \cdot\left(t(y)+\varepsilon_{1}\right) \notin \bar{D}$ for some $\varepsilon_{1}>0$. Now, define $\varphi: \bar{F} \rightarrow \bar{E}$ by $\varphi(y)=y \cdot t(y)$. It follows, from continuity of $y . t$ with respect to the initial condition $y$, that $t(y)$ and $\phi(y)$ are continuous. Now we show that $\varphi: \bar{F} \rightarrow \varphi(\bar{F})$ is a homeomorphism. By definition, $\varphi$ is one to one. So it suffices to show that, if $V$ is open in $\vec{F}$ then $\varphi(V)$ is open in $\varphi(\bar{F})$. If $V$ is open in $\bar{F}$, then $\bar{F} \backslash V$ is compact in $\bar{F}$. Hence $\varphi(\bar{F} \backslash V)$ is closed. Thus $\varphi(V)$ is open in $\varphi(\bar{F})$.

Now we show that $\varphi: \bar{F} \rightarrow \bar{E}$ is onto. To see this, note that $\varphi(\partial F)=\partial E$ and $\varphi(F)=\operatorname{int} \bar{E}$, where int $\bar{E}$ means interior of $\bar{E}$ with respect to $\partial D$. Since $F$ is open in $\partial D$, so by Brouwer's Theorem on the Invariance of Domain [9], $\varphi(F)$ must be open in $\partial D$. Thus it must be open in int $\bar{E}$ with respect to $\partial D$. On the other hand, we have $\varphi(F)=\varphi(\bar{F}) \cap \operatorname{int} \bar{E}$ and $\varphi(\bar{F})$ is closed in $\bar{E}$. Hence $\varphi(F)$ must be closed in int $\bar{E}$. Since $\varphi(F) \neq \emptyset$, it follows that $\varphi(F)=$ int $\bar{E}$. Also for $y \in \partial F$, we have $\varphi(y)=y$. It then follows that $\varphi: \bar{F} \rightarrow \bar{E}$ is onto. This means that there is a point $y_{0} \in F$ with $t\left(y_{0}\right)>0$ such that $y_{0} \cdot t\left(y_{0}\right)=\widetilde{x}$. This is impossible as $\widetilde{x}$ is a rest point of (3.1). Therefore there is a point $p \in D$ such that $p$.t is defined for all $t \geqslant 0$ and lies in $D$ for $t>0$. The $\omega$-limit set of p.t must be $\widetilde{x}$, as the flow is gradient-like and $\tilde{x}$ is the only rest point of (3.1) in $\bar{D}$. This completes the proof.

THEOREM 3.2. Suppose $f$ in (3.1) is locally Lipschitz in a neighbourhood of the closure of an open bounded set $D$ which is homeomorphic to a semisphere $\left\{x \in \mathbb{R}^{n}\right.$ : $\left.|x|<1, x_{n}>0\right\}$, and (3.1) is gradient-like with respect to a real valued function $g$ in $D$. Moreover, the following conditions hold:

$C_{1}^{\prime}$ : The set $\{x \in \bar{D}: g(x)=1-c\}$ corresponds to the set $\left\{x \in \mathbb{R}^{n}:|x| \leqslant\right.$ $\left.1, x_{n}=c\right\}$ for $0 \leqslant c \leqslant 1$, under the homeomorphism.

$C_{2}^{\prime}$ : The set $\{x \in \bar{D}: g(x)=0\}$, which consists of a single point, say $\widetilde{x}$, is a rest point of (3.1), and $\widetilde{x}$ is the only rest point of (3.1) in $\bar{D}$.

$C_{3}^{\prime}: \quad$ Let $F=\{x \in \partial D: 0<g(x)<1\}$. For $p \in \bar{F} \backslash\{\widetilde{x}\}, p . t \notin D$ for small positive $t$.

$C_{4}^{\prime}:$ For $p \in \partial D \backslash \bar{F}, p . t \in D$ for $t<0$ and small.

Then for each point $p \in \partial D \backslash \bar{F}$ we must have $\lim _{t \rightarrow \infty} p . t=\tilde{x}$.

Proof: Since $f$ is Lipschitz on $\bar{D}$ and the flow leaves $D$ as $t$ decreases, $p . t$ must be defined for all $p \in \partial D \backslash \bar{F}$ and $t \leqslant 0$. Since the $\alpha$-limit set of $p . t$ consists of a rest point, $\lim _{t \rightarrow-\infty} p . t=\tilde{x}$.

\section{Existence of TRAVElling WAves for Chapman-Jouguet detonations}

In this section we shall show that the travelling waves for Chapman-Jouguet detonation waves exist. In order to do this, we make some observations related to the nature of the stable and unstable manifold of the system (2.2) at the rest point $u_{1}$. In this direction we have the following lemma. 
LEMMA 4.1. At the rest point $u_{1}, S_{T}\left(1+p_{V}\right)-S_{V}^{2}=0$.

Proof: If we let $\varepsilon=0$ in [6, Lemma 2.4], we obtain this lemma.

The linearised system of $(2.2)$ at the rest point $u_{1}$ can be written as

$$
\dot{u}=M_{1}\left(u-u_{1}\right),
$$

where the entries of the matrix $M_{1}$ must be considered at the rest point $u_{1}$. Let $f(\lambda)$ be the characteristic polynomial of this matrix. Then by $H_{7}$, we have

$$
\begin{aligned}
f(\lambda)=\left[\lambda^{2}-\frac{1}{\alpha} V \lambda-\frac{1}{\alpha \beta} \Phi_{1}(T)\right]\left\{\lambda^{2}-\left[\frac{1}{\eta}\left(1+p_{1 V}\right)\right.\right. & \left.+\frac{1}{k} T S_{1 T}\right] \lambda \\
& \left.+\frac{T}{\eta k}\left[\left(1+p_{1 V}\right) S_{1 T}-S_{1 V}^{2}\right]\right\} .
\end{aligned}
$$

Since $V>0$ and $\Phi_{1}(T)>0$, one of the roots of the equation

$$
\lambda^{2}-\frac{1}{\alpha} V \lambda-\frac{1}{\alpha \beta} \Phi_{1}(T)=0
$$

is positive and the other one is negative. We denote these eigenvalues by $\lambda_{1}<0<\lambda_{2}$. On the other hand, from Lemma 4.1 we have

$$
\lambda^{2}-\left[\frac{1}{\eta}\left(1+p_{1 V}\right)+\frac{1}{k} T S_{1 T}\right] \lambda=0 .
$$

Thus one of the roots of the equation (4.2) is positive and the other one is zero. If we denote these eigenvalues by $\lambda_{3}=0$ and $\lambda_{4}>0$, we have proved the following theorem.

ThEOREM 4.1. Let $\lambda_{k}, 1 \leqslant k \leqslant 4$, be the eigenvalues of the matrix $M_{1}$ at the rest point $u_{1}=\left(V_{1}, T_{1}, 1,0\right)$. Then at the rest point $u_{1}, \lambda_{1}<0, \lambda_{2}>0, \lambda_{3}=0$ and $\lambda_{4}>0$.

Concerning the eigenvectors at this rest point we have the following theorem, and since its proof is similar to the proof of [3, Lemma 2.2.1] we omit the proof.

THEOREM 4.2. Let $\left(y_{1}, y_{2}, y_{3}, y_{4}\right)^{T}$ be an eigenvector corresponding to the negative eigenvalue $\lambda_{1}$. For the case of exothermic reactions, either $y_{1}<0, y_{2}>0, y_{3}>0$ and $y_{4}>0$ or the reverse inequalities hold.

In order to show the existence of travelling waves we define

and

$$
b=1+(\alpha / \beta) \sup _{\substack{G_{1}(u) \leqslant 0 \\ G_{2}(u) \geqslant 0}} \frac{\Phi_{1}(T)}{V^{2}}
$$

$$
D=\left\{u \in \mathbb{R}^{4}: G_{1}(u)<0, G_{2}(u)>0,-b<1-X+Z<0 \text { and } 0<X<1\right\},
$$

where $G_{i}(u), i=1,2$, are introduced in (2.3).

Note that the rest point $u_{1}=\left(V_{1}, T_{1}, 1,0\right)$, is located on $\partial D$. Moreover, by Theorem 4.1, the stable manifold at $u_{1}$ is one dimensional whenever this rest point exists. Concerning this manifold, we have the following lemma. 
Lemma 4.2. Let $D$ be as above. Then the stable manifold at $u_{1}$ intersects $D$ on a curve.

Proof: As shown before, the linearised system of $(2.2)$ at the rest point $u_{1}$ has the form:

$$
\dot{u}=\widetilde{M}_{1}\left(u-u_{1}\right):=\left(G_{1 L}(u), \ldots, G_{4 L}(u)\right)^{T}
$$

where $\sim$ means that the related function is considered at the rest point $u_{1}$.

Let $\left(y_{1}, y_{2}, y_{3}, y_{4}\right)^{T}$ be an eigenvector corresponding to the negative eigenvalue $\lambda_{1}$, at the rest point $u_{1}$. Now consider the solution:

$$
u(t)=(V(t), T(t), X(t), Z(t))^{T}=\left(y_{1}, y_{2}, y_{3}, y_{4}\right)^{T} e^{\lambda_{1} t}+u_{1}
$$

of the linear system (4.3). Then $u(t) \in D_{w L}$, where

$$
D_{w L}=\left\{u \in \mathbb{R}^{4}: G_{1 L}(u)<0, G_{2 L}(u)>0, G_{3 L}(u)>0 \text { and } G_{4 L}(u)>0\right\} .
$$

To see this notice that:

$\left(G_{1 L}(u), \ldots, G_{4 L}(u)\right)^{T}=\widetilde{M}_{1}\left(u-u_{1}\right)=\widetilde{M}_{1} Y e^{\lambda_{1} t}=\lambda_{1} Y e^{\lambda_{1} t}=\left(\lambda_{1} y_{1}, \ldots, \lambda_{1} y_{4}\right)^{T} e^{\lambda_{1} t}$. By Theorem $4.2, \lambda_{1}<0$, and the last equality implies that $\left(G_{1 L}(u), \ldots, G_{4 L}(u)\right)$ $\in D_{w L}$. This means that the stable manifold of (4.3), at the rest point $u_{1}$, which is the line:

$$
M_{w}=\left\{u \in \mathbb{R}^{4}: u-u_{1}=\left(y_{1}, y_{2}, y_{3}, y_{4}\right)^{T} s, s \in \mathbb{R}\right\},
$$

lies in $D_{w L}$ for $s>0$, and lies in $D$ for $s>0$ and small. Thus the stable manifold of the system (2.2) at the rest point $u_{1}$ intersects $D$ on a curve.

Now consider the following system of ordinary differential equations

$$
\begin{aligned}
\eta \dot{V} & =G_{1}(u), \\
k \dot{T} & =G_{2}(u), \\
\alpha \dot{X} & =G_{3}(u), \\
\beta V \dot{Z} & =(1-X) \Phi_{1}(T):=\widetilde{G}_{4}(u),
\end{aligned}
$$

where $G_{k}(u), 1 \leqslant k \leqslant 3$, are defined by $(2.3)$ and $\Phi_{1}(T)$ by (1.2). Notice that the above system is, mathematically, well defined for all $V>0, T>0, Z \in \mathbb{R}$ and $X \in \mathbb{R}$. Moreover it is the same as the system (2.3) for $0<X<1$ and $T>T_{i}$. This system leads us to the proof of existence of travelling waves for Chapman-Jouguet detonation waves.

Lemma 4.3. Let $D$ be as above. Then there is a unique orbit of the system (4.4) which lies in $D$, its $\omega$-limit set is $u_{1}$, and this orbit intersects the set $\Delta=\{u \in \bar{D}$ : $G_{1}(u)<0, G_{2}(u)>0$ and $\left.X=0\right\}$. Along all of these orbits $-V(t), T(t), X(t)$ and $-Z(t)$ are increasing. 
Proof: First of all note that the system (4.4) is gradient-like with respect to $h(u)=$ $X$ in $D$, and is locally Lipschitz in a neighbourhood of $\bar{D}$. We show that the system (4.4) together with $D$ (as $D$ ), $u_{1}$ (as the rest point $\widetilde{x}$ ) and the real valued function $h(u)=$ $X($ as $h)$ satisfy all of the conditions of Theorem 3.1. Conditions $C_{1}$ and $C_{2}$ hold trivially. By using lemma 4.2, Condition $C_{4}$ holds, too.

Finally, we show that Condition $C_{3}$ of Theorem 3.1 is satisfied. To see this, let $u_{0} \in\{u \in \partial D: 0<X<1\}$. Then $G_{1}\left(u_{0}\right)=0$ or $G_{2}\left(u_{0}\right)=0$ or $1-X+Z=0$ or $1-X+Z=-b$. Suppose $G_{1}\left(u_{0}\right)=0$. If we differentiate $G_{1}(u)$ along the orbits of (4.4) we obtain:

$$
\left.\frac{d G_{1}(u)}{d t}\right|_{G_{1}\left(u_{0}\right)=0}=p_{T} \frac{G_{2}(u)}{k}-\left.p_{X} \frac{V}{\alpha}(1-X+Z)\right|_{u=u_{0}}>0 .
$$

Thus the flow goes out of $\bar{D}$ on $G_{1}\left(u_{0}\right)=0$. Similarly, let $G_{2}\left(u_{0}\right)=0$ and differentiate $G_{2}(u)$ along the orbits to obtain:

$$
\left.\frac{d G_{2}(u)}{d t}\right|_{G_{2}\left(u_{0}\right)=0}<0
$$

Hence, the flow goes out of $\bar{D}$ on $G_{2}\left(u_{0}\right)=0$. If we differentiate $\widetilde{G}_{3}(u)=1-X+Z$ along the orbits we get:

$$
\begin{aligned}
& \left.\frac{d \widetilde{G}_{3}(u)}{d t}\right|_{1-X+Z=0}=\frac{1-X}{\beta V} \Phi_{1}(T)>0, \\
& \left.\frac{d \widetilde{G}_{3}(u)}{d t}\right|_{1-X+Z=-b}=-b \frac{V}{\alpha}+\frac{1-X}{\beta V} \Phi_{1}(T)<0 .
\end{aligned}
$$

Therefore, the flow goes out of $\bar{D}$ on $\widetilde{G}_{3}\left(u_{0}\right)=0$ and $\widetilde{G}_{3}\left(u_{0}\right)=-b$. Hence Condition $C_{3}$ of Theorem 3.1 holds too. Thus by Theorem 3.1, there must be an orbit of the system (4.4) lying in $D$, starting at a point on the surface $X=0$ and running to the point $u_{1}$ as $t \rightarrow+\infty$. Finally, from the system (4.4) and the set $D$ it follows that along this orbit $X(t)$ and $T(t)$ are increasing, but $V(t)$ and $Z(t)$ are decreasing.

Let $\widetilde{u}(t), t \in\left[t_{0}, \infty\right)$ be the orbit which is given by the above lemma. Then $\widetilde{u}\left(t_{0}\right) \in$ $\{u \in \bar{D}: X=0\}$ and $\lim _{t \rightarrow \infty} \tilde{u}(t)=u_{1}$. Concerning the orbit $\tilde{u}(t)$ we have the following lemma.

LEMMA 4.4. Let $\tilde{u}(t)$ be as above. Then there is a number a such that for all $(\eta, k, \alpha, \beta)>0$ with $\min (\alpha, \beta)>\max (k, \eta)$, the orbit $\tilde{u}(t)$ meets the hypersurface $T=T_{i}$ for some $\tilde{t} \in\left(t_{0}, \infty\right)$, with $0<\tilde{X}(\tilde{t})<1$ and $-1<\tilde{Z}(\tilde{t})<0$.

PROOF: Let $u_{01}=\left(V_{01}, T_{01}, 0,-1\right)^{T}$ and $u_{00}=\left(V_{00}, T_{00}, 0,-1\right)^{T}$, where $\left(V_{01}, T_{01}\right)$ and $\left(V_{00}, T_{00}\right)$ are introduced by Lemma 2.2 , and $u_{1}$ is as above. Choose a positive number $a$ so large such that the points $u_{00}$ and $u_{01}$ are on different sides of the hypersurface $P:\left(T-T_{1}\right)-a\left(V-V_{1}\right)=0$, where $V_{1}$ and $T_{1}$ are the first and second components of $u_{1}$. This is possible as $V_{01}<V_{1}<V_{00}$. 
Let $\left(V_{i}, T_{i}, X_{i}, Z_{i}\right)^{T}$ be the unique solution of the equations

$$
G_{1}(u)=0, G_{2}(u)=0,1-X+Z=0 \text { and } T=T_{i}
$$

Then from $T_{00}<T_{i}<T_{1}$, it follows that $0<X_{i}<1,-1<Z_{i}<0,\{u \in \bar{D}$ : $G_{1}(u)=0, G_{2}(u)=0,0<X<X_{i}$ and $\left.-1<Z<Z_{i}\right\} \subset\left\{u \in \bar{D}: T \leqslant T_{i}\right\}$, and $V_{1}<V_{i}<V_{00}$. Now consider the hypersurface $P^{\prime}:\left(T-T_{i}\right)-a\left(V-V_{i}\right)=0$. Since $u_{00}=\left(V_{00}, T_{00}, 0,-1\right)^{T} \in\left\{u \in \bar{D}:\left(T-T_{i}\right)-a\left(V-V_{i}\right)<0\right\}$, we can choose $0<X_{0}<X_{i}$ and $-1<Z_{0}<Z_{i}$ such that $\left\{u \in \bar{D}: G_{1}(u)=0, G_{2}(u)=0,0<X<X_{0}\right.$ and $\left.-1<Z<Z_{0}\right\} \subset\left\{u \in \bar{D}:\left(T-T_{i}\right)-a\left(V-V_{i}\right)<0\right\}$. Let $D_{0}=\left\{u \in D: T_{i}-a V_{i}<\right.$ $\left.T-a V<T_{1}-a V_{1}, 0<X<X_{0}\right\} \cup\left\{u \in D: T_{i}-a V_{i}<T-a V<T_{1}-a V_{1},-1<Z<Z_{0}\right\}$ and $\delta=\min _{u \in \bar{D}_{0}}\left[G_{2}(u)-a G_{1}(u)\right]$. Then $\delta>0$.

Now suppose the orbit $\tilde{u}(t), t \in\left[t_{0},+\infty\right)$, does not meet the set $\{u \in D: T=$ $T_{i}, 0<X<1$ and $\left.-1<Z<0\right\}$. Let $t_{1}>t_{0}$ and $t_{2}>t_{3}>t_{0}$ be the solutions of the equations $\tilde{X}(t)=X_{0}, \widetilde{Z}(t)=Z_{0}$ and $\widetilde{Z}(t)=-1$, respectively. Here $\widetilde{X}(t)$ and $\widetilde{Z}(t)$ are the third and fourth components of $\widetilde{u}(t)$, respectively. Since $\frac{d}{d t}[T-a V]=$ $(1 / k) G_{2}(u)-(a / \eta) G_{1}(u)>0, T-a V$ is increasing along the orbit $\tilde{u}(t)$. It follows that $\widetilde{u}(t)$ remains in $D_{0}$ for $\max \left(t_{2}, t_{1}\right)>t>t_{0}$. Now along the orbit $\widetilde{u}(t)$ in $D_{0}$ we have:

$$
\begin{aligned}
\frac{d}{d X}[T-a V] & =\frac{1}{\frac{d X}{d t}}\left[\frac{d T}{d t}-a \frac{d V}{d t}\right] \\
& =\frac{\alpha}{G_{3}(u)}\left[\frac{G_{2}(u)}{k}-a \frac{G_{1}(u)}{\eta}\right] \\
& \geqslant \frac{\alpha \delta \sigma}{\max (k, \eta)}>0,
\end{aligned}
$$

where $1 / \sigma=\max _{u \in \bar{D}} G_{3}(u)$. Similarly,

$$
\frac{d}{d Z}[T-a V] \geqslant \frac{\beta \delta \varepsilon}{\max (k, \eta)}>0,
$$

where $1 / \varepsilon=\max _{u \in \bar{D}}(1-X / V) \Phi_{1}(T)$. Let $T_{0}=T\left(t_{0}\right), V_{0}=V\left(t_{0}\right)$. Then $T_{0}-a V_{0}<T_{i}-a V_{i}$, if $\tilde{u}(t)$ does not meet $T_{i}$. Therefore

$$
\begin{aligned}
\left(T_{1}-a V_{1}\right)-\left(T_{i}-a V_{i}\right) & \geqslant \int_{t_{0}}^{\infty}\left[\frac{1}{k} G_{2}(\tilde{u}(t))-\frac{a}{\eta} G_{1}(\tilde{u}(t))\right] d t \\
& \geqslant \int_{t_{0}}^{t_{1}}\left[\frac{1}{k} G_{2}(\tilde{u}(t))-\frac{a}{\eta} G_{1}(\tilde{u}(t))\right] d t \\
& =\int_{0}^{X_{0}} \frac{\alpha}{G_{3}(u)}\left[\frac{G_{2}(u)}{k}-a \frac{G_{1}(u)}{\eta}\right] d X \geqslant \frac{\alpha \delta \sigma X_{0}}{\max (k, \eta)}
\end{aligned}
$$


Similarly,

$$
\begin{aligned}
\left(T_{1}-a V_{1}\right)-\left(T_{i}-a V_{i}\right) & \geqslant \int_{t_{3}}^{t_{2}}\left[\frac{1}{k} G_{2}(\widetilde{u}(t))-\frac{a}{\eta} G_{1}(\widetilde{u}(t))\right] d t \\
& \geqslant \int_{-1}^{Z_{0}} \frac{\beta V}{(1-X) \Phi_{1}(T)}\left[\frac{G_{2}(u)}{k}-a \frac{G_{1}(u)}{\eta}\right] d Z \geqslant \frac{\beta \delta \varepsilon\left(Z_{0}+1\right)}{\max (k, \eta)} .
\end{aligned}
$$

Note that the first inequality is impossible for $\alpha \gg \max (k, \eta)$ and the second inequality is impossible for $\beta \gg \max (k, \eta)$. Thus such a positive number $a$ and $\widetilde{t}>t_{0}$ exist.

From now on we assume that $\min (\alpha, \beta) \gg \max (k, \eta)$, so the orbit $\tilde{u}(t)$ meets the surface $T=T_{i}$ at the point $\widetilde{u}_{i}=\left(\widetilde{V}_{i}, \widetilde{T}_{i}, \widetilde{X}_{i}, \widetilde{Z}_{i}\right)^{T}$, where $V_{1}<\widetilde{V}_{i}<V_{00}, \widetilde{T}_{i}=T_{i}, 0<$ $\widetilde{X}_{i}<1$ and $-1<\widetilde{Z}_{i}<0$. We call the point $\tilde{u}_{i}$ the ignition point. According to Lemma 4.2 , this point, is unique for Chapman-Jouguet detonation.

Now we have our main theorem.

THEOREM 4.3. Suppose that the system (2.2) admits the rest points $u_{1}$ and $u_{m 0}$, for some $0 \leqslant m<1$. If Conditions (1.3)-(1.4) and Hypotheses $H_{1}-H_{8}$ hold, then for given $\eta, k, \alpha, \beta>0$ with $\min (\alpha, \beta) \gg \max (\eta, k)$, there is a unique orbit of the system (2.2) which runs from $u_{m 0}$ to $u_{1}$, for some $0 \leqslant m<1$.

Proof: In the region $T<T_{i}$, the last equation of (2.2) becomes $\dot{Z}=0$. Thus, in this region, along the orbits of this system $Z(t)$ is constant. Here we let $Z(t)=\bar{Z}_{i}$, where $\widetilde{Z}_{i}$ is the fourth component of $\widetilde{u}_{i}$, the ignition point. On the surface $Z=\widetilde{Z}_{i}$, the system (2.2) reduces to the following three dimensional system of equations, in the region $T \leqslant T_{i}:$

$$
\begin{aligned}
\eta \dot{V} & =V-J+p(V, T, X)=F_{1}(V, T, X), \\
k \dot{T} & =e(V, T, X)-\frac{1}{2} V^{2}+J V-q(V, T)\left(1-X+\widetilde{Z}_{i}\right)=F_{2}(V, T, X), \\
\alpha \dot{X} & =-V\left(1-X+\widetilde{Z}_{i}\right)=F_{3}(V, T, X) .
\end{aligned}
$$

Now consider the region $D^{\prime}=\left\{(V, T, X) \in \mathbb{R}^{3}: F_{1}(V, T, X)<0, F_{2}(V, T, X)\right\rangle$ $0,1-X+\widetilde{Z}_{i}<0$ and $\left.T-V+X<\widetilde{T}_{i}-\widetilde{V}_{i}+\widetilde{X}_{i}\right\}$. Notice that $\widetilde{T}_{i}=T_{i}$ and $\left(\widetilde{V}_{i}, \widetilde{T}_{i}, \widetilde{X}_{i}\right) \in$ $\partial D^{\prime}$. By using Theorem 3.2, we show that any orbit of (4.5) initiating at a point on $\partial D^{\prime} \cap\left\{(V, T, X): T-V+X=\widetilde{T}_{i}-\widetilde{V}_{i}+\widetilde{X}_{i}\right\}$ approaches the unique rest point of system (4.5) which is located in the region $T<T_{i}$, as $t$ tends to $-\infty$. We denote this rest point by $\left(\bar{V}_{i}, \bar{T}_{i}, \bar{X}_{i}\right)$. In order to do this we show that the open set $D^{\prime}$, the rest point $\left(\bar{V}_{i}, \bar{T}_{i}, \bar{X}_{i}\right)$ and the function $g(V, T, X)=\left(T-\bar{T}_{i}-V+\bar{V}_{i}+X-\bar{X}_{i}\right) /\left(\widetilde{T}_{i}-\bar{T}_{i}-\widetilde{V}_{i}+\bar{V}_{i}+\widetilde{X}_{i}-\bar{X}_{i}\right)$ satisfy all of the conditions of Theorem 3.2. It is easy to see that conditions $C_{1}^{\prime}$, and $C_{2}^{\prime}$ are satisfied. Also if we differentiate $F_{1}, F_{2}$, and $g$ along the orbits of (4.5) on the boundary $D^{\prime}$, then the flow goes out of $D^{\prime}$ on $\left\{(V, T, X): F_{1}=0\right\} \cup\left\{(V, T, X): F_{2}=\right.$ $0\} \cup\{(V, T, X): g=1\}$. Moreover $\partial D^{\prime} \cap\left\{(V, T, X): F_{3}(V, T, X)=0\right\}$ is invariant with respect to the system (4.5). Hence Condition $C_{3}^{\prime}$ is satisfied too. Therefore by Theorem 
3.2 , each orbit of (4.5) starting at a point of $\partial D^{\prime} \cap\left\{(V, T, X): T-V+X=\widetilde{T}_{i}-\widetilde{V}_{i}+\widetilde{X}_{i}\right\}$ lies in $\overline{D^{\prime}}$ for $t<0$ and goes to $\left(\bar{V}_{i}, \bar{T}_{i}, \bar{X}_{i}\right)$ as $t$ tends to $-\infty$. Note that along this orbit, $-V(t), T(t)$ are increasing and $X(t)$ is nondecreasing.

Now, consider again the ignition point $\widetilde{u}_{i}=\left(\tilde{V}_{i}, \widetilde{T}_{i}, \tilde{X}_{i}, \widetilde{Z}_{i}\right)$. By the above argument, there is a unique orbit of the system $(2.2)$, say $\widetilde{\widetilde{u}}(t)=(\tilde{\widetilde{V}}(t), \widetilde{\widetilde{T}}(t), \widetilde{\widetilde{X}}(t), \widetilde{\widetilde{Z}}(t)),-\infty<$

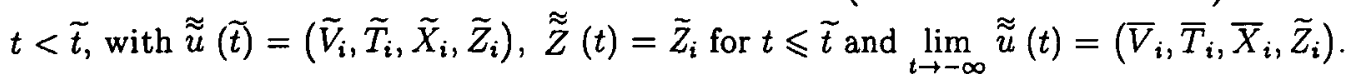
Along this orbit $-V(t), T(t)$ and $X(t)$ are increasing and $Z(t)$ is constant. This orbit lies in $D$, the domain which is used in the proof of Lemma 4.3. Now define

$$
u(t)= \begin{cases}\widetilde{u}(t) & t \geqslant \tilde{t} \\ \widetilde{\tilde{u}}(t) & t<\tilde{t}\end{cases}
$$

Then $u(t)$ is a complete orbit of the system (2.2) lying in $D$ and runs from $u_{m 0}$ to $u_{1}$ for some $0 \leqslant m<1$. This completes the proof.

\section{REFERENCES}

[1] R. Gardner, 'On the detonation of a combustible gas', Trans. Amer. Math. Soc. 277 (1983), 431-468.

[2] Z. Gasser and P. Szmolyan, 'A geometric singular perturbation analysis of detonation and deflagration waves', SIAM J. Math. Anal. 24 (1993), 968-986.

[3] M. Hesaaraki, 'The structure of shock waves in magnetohydrodynamics', Mem. Amer. Math. Soc. 302 (1984).

[4] M. Hesaaraki, 'The structure of shock waves in magnetohydrodynamics for purely transverse magnetic fields', SIAM J. Appl. Math. 51 (1991), 412-428.

[5] M. Hesaaraki, 'The structure of MFD shock waves in a model of two fluids', Nonlinearity 6 (1993), 1-24.

[6] M. Hesaaraki, 'The structure of MFD shock waves', Pures. Appl. 72 (1993), 377-404.

[7] M. Hesaaraki, 'The structure of MFD shock waves for rectilinear motion in some models of plasma', Trans. Amer. Math. Soc. 347 (1995), 3423-3452.

[8] J.A. Smoller, Shock waves and reacting diffusion equations (Springer-Verlag, Berlin, Heidelberg, New York, 1994).

[9] J.W. Vick, Homology theory. An introduction to algebraic topology, Pure and Appl. Math. 53 (Academic press, Inc., New York, London, 1973).

[10] D. Wagner, 'The existence and behavior of viscous structure for plane detonation waves', SIAM J. Math. Anal. 20 (1989), 1035-1054.

[11] F.A. Williams, Combustion theory (Benjamin/Cummings, Menlo Park, CA, 1985).

Department of Mathematics

Sharif University of Technology

P.O. Box 11365-9415

Tehran

Iran

e-mail: hesaraki@math.sharif.ac.ir

\author{
Department of Mathematics, \\ Faculty of Science, \\ Tarbiat Modarres University, \\ P.O. Box 14155-4838, \\ Tehran \\ Iran \\ e-mail: razani@karun.ipm.ac.ir \\ detwave@vax.ipm.ac.ir
}

